\title{
Über den Widerstand und die Kapazität an den Grenzflächen elektrochemischer Elektroden
}

\author{
Von Georg Falk und Erich Lange \\ Aus dem Physikalisch-Chemischen Laboratorium der Universität Erlangen
}

(Z. Naturforschg. 1, 388-392 [1946]; eingegangen am 16. April 1946)

\begin{abstract}
An elektrochemischen Elektroden zwischen Metallphase und Elektrolyt vorhandene Grenzschichten verursachen im Stromfluß einen als Widerstandspolarisation bezeichneten Anteil der Gesamtpolarisation. Bei hohem spezifischem Widerstand ist der Aufbau des $\mathrm{Ohm}$ schen Spannungsgefälles mit Raumladungen und daher mit merklichen Anlauf- und Abklingzeiten verknüpft. Bei sehr kurzen Strombelastungen, insbesondere im Wechselstrom hoher Frequenz, macht sich dies wie ein Wirk- bzw. Blindwiderstand bemerkbar. Die Messungen an den Systemen $\mathrm{Cu} / \mathrm{Cu}$-Ionenlösung und $\mathrm{Pb} / 4-m$. Schwefelsäure ergaben Widerstände von $1,5 \mathrm{Ohm} \cdot \mathrm{cm}^{2}$ bzw. $5,5 \mathrm{Ohm} \cdot \mathrm{cm}^{2}$. Die zum Teil unerwartete Größe hat verschiedene theoretisch interessante und praktisch wichtige Bedeutung.
\end{abstract}

\section{Problemstellung}

Bei elektrochemischen Elektroden kann eine zwischen Metallphase und Elektrolyt vorhandene, u. U. sonst kaum nachweisbare, schlechtleitende Schicht im stationären Stromfluß ein Ohmsches Spannungsgefälle bewirken, das als Widerstandspolarisation bezeichnet und gemessen wird. Bei einer solch dünnen Widerstandsschicht von hohem spezifischen Widerstand sind die zum Aufbau des Ohmschen Spannungsgefälles benötigten Raumladungen nicht zu vernachlässigen; daher benötigt die Einstellung des stationären $\mathrm{O}$ h m schen Spannungsgefälles bei Stromschluß und das Verschwinden desselben nach Stromöffnung, etwa wie eine Parallelschaltung von Kondensator und $\mathrm{Ohm}$ schem Widerstand, endliche Zeit. Im folgenden soll nun versucht werden, von der an einer einfachen elektrochemischen Elektrode im Stromfluß auftretenden, im wesentlichen auf Konzentrations-, Aktivierungs- und Widerstandspolarisation beruhenden Gesamtpolarisation die Widerstandspolarisation $^{1}$ und den Kapazitätseinfluß mit geeigneten experimentellen Mitteln abzutrennen. Bisweilen kann zwar während und infolge der elektrolytischen Stoffumsetzungen an einer anfänglich einfachen Elektrode zu der zugehörigen einen potentialbestimmenden Ionenart, wie bei einer mehrfachen Elektrode, noch eine andere hinzukommen oder

1 Eine ausführliche Literaturzusammenstellung findet sich in: Gmelins Handb. d. anorgan. Chemie, 8. Aufl., Verlag Chemie Berlin 1942, Syst.-Nr.68 Pt Tl. B, Lieferung 3, S. 200 u. S. 226. gar vorherrschend werden, wodurch eine Änderung des Ruhegalvanipotentials bewirkt würde. Diese sog. Abscheidungspolarisation dürfte aber im folgenden, dank der Wahl des Systems und der Untersuchungsmethode, keine Rolle spielen.

\section{Theoretische Gesichtspunkte zur Untersuchungsmethode}

Verändert man beispielsweise die Dauer der Polarisierung einer elektrochemischen Elektrode bei konstantem Strom, so wird sich im allgemeinen auch der Wert der an einer Elektrode auftretenden Polarisation

$$
\Delta \Delta \varphi=\Delta \varphi_{\text {Strom }}-\Delta \varphi_{\text {Ruhe }^{2}},
$$

der eine Funktion der Zeit und der geflossenen Elektrizitätsmenge ist, verändern. Man kann aber von der Annahme ausgehen, daß die Widerstandspolarisation, ungeachtet ihrer erforderlichen Anlaufzeit, schneller als die anderen Polarisationsarten zur Ausbildung kommt, so daß die bei sehr kurzen Polarisierungszeiten meßbare Gesamtpolarisation praktisch nur Widerstandspolarisation darstellt und noch keine anderen Polarisationsarten enthält.

Eine bequeme Methode zur Variation und Herabsetzung der Polarisierungszeit bietet die Verwendung von Wechselströmen hoher Frequenz.

2 Uber die verschiedenen Potentiale an elektrochemischen Systemen s. bei E. L a n ge, Handb. d. Experimentalphysik XII, 2. Tl.; II Elektrochemie, Tl. VII Elektrochemie der Phasengrenzen, S. $265 \mathrm{ff}$. 
Hierbei werden sich reversible Anteile der Polarisation als sog. Blindwiderstand $R_{b}=1 / \omega C=P / \omega$ $=d E / d q \cdot 1 / \omega$ und irreversible Anteile als Wirkwiderstand $R_{w}=N / J^{2}$ bemerkbar machen, wobei $C$ die fiktive Polarisationskapazität, $P=d E / d q$ die Polarisierbarkeit und $N$ den Leistungsverlust beim Strom $J_{\text {eff }}$ darstellen. Vollständig reversibel wäre die Gesamtpolarisation einer Elektrode, wenn die für die Entstehung und Aufrechterhaltung einer bestimmten Polarisation $\Delta \Delta \varphi$ während der Zeit $t$ bei konstanter Stromdichte $i$ aufzuwendende Polarisationsarbeit $i \cdot \Delta \Delta \varphi \cdot t$ zahlenmäßig gerade so groß wäre wie die Depolarisationsarbeit, die erforderlich ist, damit die nachfolgende Umkehrung desselben Stromes $i$ während der gleichen Zeit $t$ gerade ein Wiederverschwinden von $\Delta \Delta \varphi$ zur Folge hat. Jede Abweichung hiervon beruht auf irreversiblen Polarisationsanteilen, die gemessen werden an Hand eines im Zeitmittel auftretenden irreversiblen Arbeitsverbrauches oder der Wärmeentwicklung. So wie jede zu unterscheidende Grundpolarisationsart läßt sich auch die Widerstandspolarisation in einen solchen reversiblen und in einen irreversiblen Anteil aufteilen. Bei ihr ist ersterer durch das rückwärtige Wiederabfließen der in der Widerstandsschicht angehäuften Raumladungen, letzterer durch den irreversiblen Stromfluß und die Joulesche Wärme in dieser Schicht bedingt.

\section{Praktische Gesichtspunkte zur Me- thode}

Die praktische Untersuchung der Widerstands- und Kapazitätseigenschaften der Grenzflächenschicht einer Elektrode im Rahmen einer ganzen Zelle wurde mit der Wechselstrombrücke durchgeführt. Als Versuchsgefä $\beta$ diente ein zylindrisches Leitfähigkeitsgefäß aus Trolitul mit auswechselbaren Elektroden von gleichem Querschnitt wie die zylindrische Höhlung. Die Elektroden, die auf der Stirnfläche von Trolitulzylindern $Z$ (Abb. 1) sitzen, konnten konzentrisch, stabil und dicht von beiden Seiten her in das Versuchsgefäß eingeschoben werden. Verschiedene, willkürlich wählbare und ausreichend reproduzierbare meßbare Entfernungen zwischen den Elektrodenoberflächen konnten dadurch eingestellt werden, daß zunächst die Elektroden bis zur völligen Berührung zusammengeschoben wurden. Dann wurde die eine Elektrode nach Heranschieben des Metallstückes $M$ an die geschliffene Stirnfläche des Trolituldeckels $D$ und Festschrauben auf der konzentrischen Stromzuführung $A$ so weit wieder nach außen verschoben, daß die Lücke zwischen der Anfangs- und der Endstellung des Metallstückes $M$ gerade durch eine bekannte Metall-Lehre $L$ ausgefüllt wurde. Zwei in die Trolitulwand gegenüber- stehend einmündende Löcher von $1 \mathrm{~mm} \varnothing$ gestatteten die Einführung und ständige Erneuerung des benutzten Elektrolyten, der vorher nach Durchfließen einer Glasrohrschlange in einem Thermostaten auf konstante Temperatur gebracht worden war. Da die öffnung, durch die der Elektrolyt einfloß, schräg gebohrt war, konnte eine gute Durchwirbelung des Elektrolyten während der Messung gewährleistet werden. Die Meßbrücke ${ }^{3}$ selbst bestand aus zwei gleichen Widerständen von $100 \mathrm{Ohm}$, die an einem Ende aneinandergelötet waren. Ihre freien Enden waren mit den Ausgangsbuchsen eines symmetrischen Schwebungssummers fest verbunden, während ihre Lötstelle zum Nullzweig der Brücke führte. Der Nullstrom wurde über einen Verstärker dem Nullinstru-

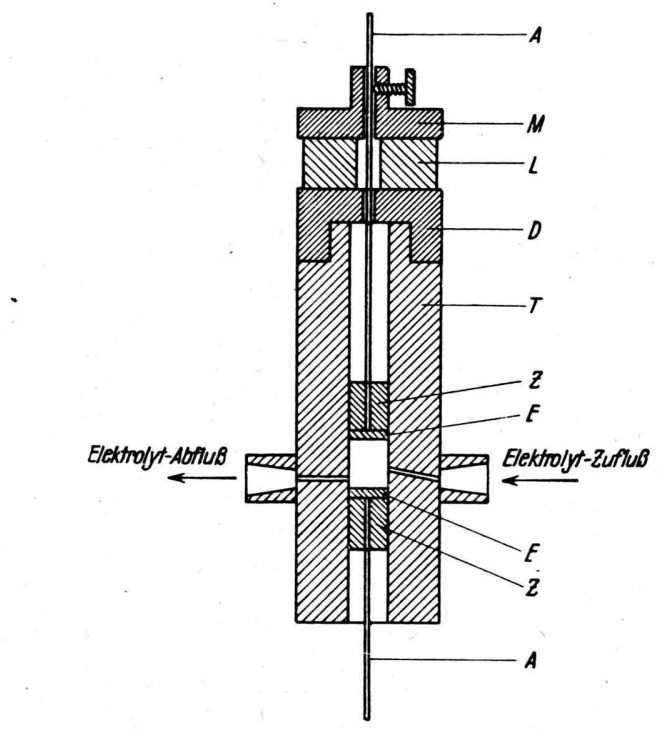

Abb. 1. Versuchsgefäß im Schnitt. $A$ Kupferstäbe $2 \mathrm{~mm} \varnothing$ als Stromzuleitung, $M$ Metallstück mit Rändelschraube, $L$ Metall-Lehre, $D$ Trolituldeckel, $T$ Trolitulrohr mit Bohrung, $Z$ Trolitulzylinderchen, $E$ Elektroden.

ment zugeleitet. Als solches diente im Bereich von 1000 bis $4000 \mathrm{~Hz}$ ein Telephon, bei höheren Frequenzen ein mittels Parallelschwingkreis selektiv gemachtes Röhrenvoltmeter. Zum Abgleich der Brücke benützten wir im Vergleichszweig einen Kurbeldekadenwiderstand von 0,1 bis $1111 \mathrm{Ohm}$ mit Wicklung nach Wagner und Wertheimer und einen Stöpselrheostaten von 0,1 bis $111111 \mathrm{Ohm}$ mit Chaperon-Wicklung sowie einen Drehkondensator bis $1000 \mathrm{~cm}$ und einen Kurbeldekadenkondensator von 0,001 bis $1,11 \mu F$. Weitere einzelne Kondensatoren konnten hierzu im Bedarfsfalle parallel geschaltet werden.

Die Ermittlung des Wirkwiderstandes des Elektrolyten sowie des Blindwiderstandes der Erdkapazität und der geometrischen Kapazität der sich gegenüberstehen-

3 Ausführliche Literaturangaben, die wesentlichen Teile derartiger Brückenschaltungen betreffend, finden sich z. B. im Handb. d. Experimentalphysik XIV, 1, Leitfähigkeit in flüssigen Elektrolyten 2. Kap. Die Messung der Leitfähigkeit von flüssigen Elektrolyten. S. 16 ff. 
den Elektroden erfolgte mit zwei platinierten Platinelektroden im gleichen Gefäß, bei deren Verwendung sich der komplexe Widerstand der gesamten Leitfähigkeitszelle erfahrungsgemäß lediglich aus dem Wirkwiderstand des Elektrolyten und dem Blindwiderstand der geometrischen und der Erdkapazität zusammensetzt. Hierauf wurde die eine der beiden platinierten Platinelektroden durch eine zu untersuchende Elektrode bei gleichem Elektrodenabstand ersetzt; der vorher ermittelte komplexe Widerstand der Zelle blieb im Vergleichszweig eingestellt, während die unbekannten Beträge von $R$ und $C$ bei stets gutem Minimum bestimmt wurden. Es liegt zur rechnerischen Vereinfachung nahe, als Vergleich jeweils eine Serienschaltung von $\mathrm{Ohm}$ schem Widerstand und Kondensator $\mathrm{zu}$ verwenden ${ }^{4}$; denn dann ist der resultierende Wirkwiderstand dieser Ersatzschaltung gleich dem Wert ihres rein $\mathrm{Oh}$ m schen

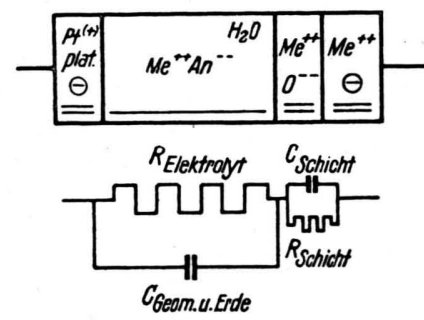

Abb. 2. Ersatzschaltbild einer Leitfähigkeitszelle, mit einer plat. Platinelektrode.

Widerstandes, während sich ihr resultierender Blindwiderstand in einfacher Weise aus der Kapazität $C$ des Kondensators nach $1 / \omega c$ errechnet. Entsprechend der wahrscheinlichen Natur der Grenzflächenschichten (Abbildung 2) wurde jedoch zum Vergleich eine Parallelschaltung von $\mathrm{Ohm}$ schem Widerstand und Kondensator benützt.

\section{Experimentelle Ergebnisse}

In Abb. 3 und 4 sind die an den Systemen $\mathrm{Cu} / \mathrm{Cu}$ Ionenlösung und $\mathrm{Pb} / 4-\mathrm{m}$. Schwefelsäure gewonnenen und in Tab. I und II angeführten Meßergebnisse, vor allem in Form von $R$ und $C$ der angenommenen Elektrodenschichten, alle in Abhängigkeit von der Meßfrequenz eingetragen. Wir nehmen an, daß der oberhalb $12000 \mathrm{~Hz}$ innerhalb der Meßgenauigkeit konstant gefundene Wirk- und Blindwiderstand auf eine Grenzflächenschicht von

4 Siehe hierzu F، End e r. Zur Methodik der elektrolytischen Leitfähigkeitsmessung, Z. Elektrochem. 43, 217 [1937].

5 Ähnliche experimentelle Ergebnisse bezüglich merklicher Widerstands- und Kapazitätseigenschaften von Elektrodengrenzflächen s. bei P. Dolin u. B. Ershele r, Kinetics of Processes on the Platinum Electrode. Acta physicochim. URSS XIII, 747 ff. [1940]. Die dortigen Befunde werden im Sinne einer Aktivierungspolárisation gedeutet, während die Möglichkeit eines bestimmtem Widerstand und bestimmter Kapazität zurückzuführen ist und kein merklicher Einfluß einer etwaigen Aktivierungspolarisation hereinspielt $^{5}$. Die bei niederen Frequenzen wie $12000 \mathrm{~Hz}$ auftretenden größeren Werte von $C$ und $R$ sind offenbar auf andere, langsamer entstehende, reversible und irreversible Polarisationsarten zurückzuführen.

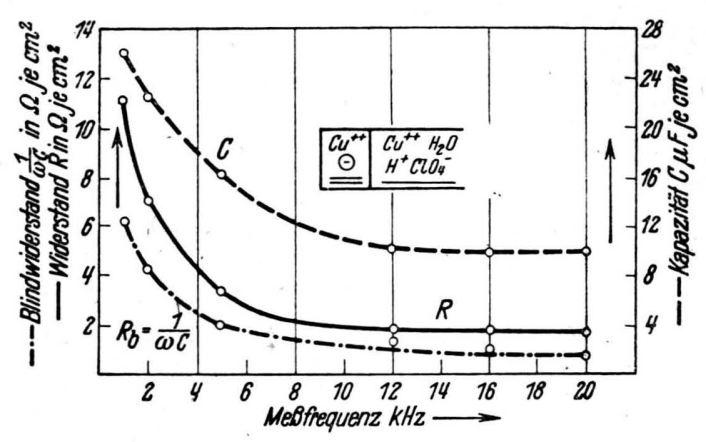

Abb. 3. Ergebnis der Brückenmessung am System $\mathrm{Cu} / \mathrm{Cu}++. C_{\mathrm{Cu}^{++}}=m / 1 \cup 0, C_{\mathrm{H}^{+}}=m / 1000$.

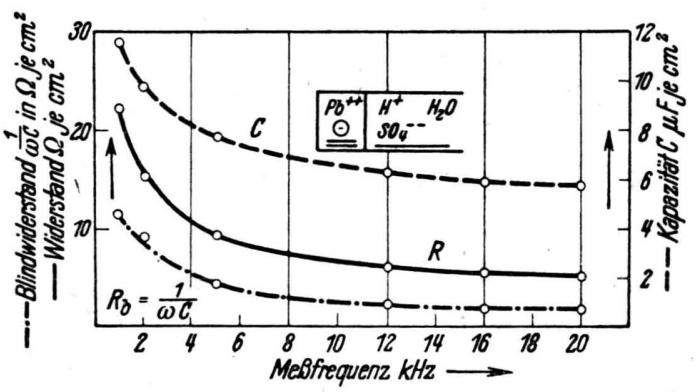

Abb. 4. Ergebnis der Brückenmessung am System $\mathrm{Pb} / \mathrm{H}_{2} \mathrm{SO}_{4} . C_{\mathrm{H}}+=m / 100$.

\section{Diskussion der Ergebnisse}

Aus dem experimentellen Ergebnis lassen sich nun einige wichtige Tatsachen entnehmen. $\mathrm{Zu}-$ nächst erscheint die Größe des Widerstandes einer Grenzflächenschicht am System Pb/4-m. Schwefelsäure wegen der an diesem System bekannten und sichtbaren Bildung einer Bleisulfatschicht von ca. $5,5 \mathrm{Ohm} \cdot \mathrm{cm}^{2}$ vielleicht nicht unerwartet. Dagegen dürfte am System $\mathrm{Cu} / \mathrm{Cu}$-Ionenlösung die Anwe-

entsprechenden Schichtwiderstandseinflusses nicht in Betracht gezogen wird. Wir neigen, wie erwähnt, zu der Auffassung, daß ein solcher aus verschiedenen Gründen nicht zu vernachlässigen ist. Allerdings wird schließlich jeder Widerstand einer Schicht, insbesondere von atomarer Dicke, im Sinne zu überwindender Ablösearbeiten aufzufassen sein. Siehe hierzu z. B. Handb. d. Experimentalphysik XII, 1; Tl. III, Leitfähigkeit und Ưberführungszahlen in festen Elektrolyten, S. $435 \mathrm{ff}$. 


\begin{tabular}{|c|c|c|c|c|c|c|c|}
\hline \multirow{3}{*}{$\begin{array}{c}\text { Meß- } \\
\text { frequenz } f \\
\text { in } \mathrm{kHz}\end{array}$} & \multicolumn{2}{|c|}{$\begin{array}{l}\text { Parallelwiderstand } R \\
\text { in } \Omega \mathrm{x} \mathrm{cm}^{2}\end{array}$} & \multicolumn{2}{|c|}{$\begin{array}{c}\text { Parallelkapazität } C \\
\text { in } \mu F / \mathrm{cm}^{2}\end{array}$} & \multirow{3}{*}{$\begin{array}{c}\text { Blindwider- } \\
\text { stand } 1 / \omega C \\
\text { des Parallel- } \\
\text { kondensators } \\
\text { in } \Omega \mathrm{x} \mathrm{cm}^{2}\end{array}$} & \multirow{3}{*}{$\begin{array}{c}\text { Resultierender } \\
\text { Wirkwiderstand } \\
\text { der Parallel- } \\
\text { schaltung } R_{w}= \\
R /\left(1+\omega^{2} R^{2} C^{2}\right) \\
\text { in } \Omega \times \mathrm{cm}^{2} \\
\end{array}$} & \multirow{3}{*}{$\begin{array}{c}\text { Resultierender } \\
\text { Blindwiderstand } \\
\text { der Parallel- } \\
\text { schaltung } R_{b}= \\
R^{2} \omega C /\left(1+\omega^{2} R^{2} C^{2}\right) \\
\text { in } \Omega \times \mathrm{cm}^{2}\end{array}$} \\
\hline & \multirow{2}{*}{$\begin{array}{c}\text { Mittel- } \\
\text { wert }\end{array}$} & \multirow{2}{*}{$\begin{array}{c}\text { Mittlerer Fehler } \\
\text { des Mittels } \\
F_{m}=\sqrt{\frac{\Sigma \Delta^{2}}{n(n-1)}}\end{array}$} & \multirow{2}{*}{$\begin{array}{l}\text { Mittel- } \\
\text { wert }\end{array}$} & \multirow{2}{*}{$\begin{array}{l}\text { Mittlerer Fehler } \\
\text { des Mittels } \\
F_{m}=\sqrt{\frac{\sum \Delta^{2}}{n(n-1)}}\end{array}$} & & & \\
\hline & & & & & & & \\
\hline 1 & 11 & $\pm 1,1$ & 26 & $\pm 1,5$ & 6,1 & 2,5 & 4,6 \\
\hline 2 & 7 & $\pm 0,44$ & 19 & $+1,5$ & 4,2 & 1,8 & 3,1 \\
\hline$\overline{5}$ & 3,3 & $\pm 0,21$ & 16 & $\pm 1,1$ & 2,0 & 0,9 & 1,5 \\
\hline 12 & 1,7 & $\pm 0,13$ & 10 & $\pm 1,1$ & 1,3 & 0,6 & 0,9 \\
\hline 16 & 1,7 & $\pm 0,17$ & 10 & $\pm 0,71$ & 1 & 0,44 & 0,8 \\
\hline 20 & 1,5 & $\pm 0,14$ & 11 & $\pm 1,3$ & 0,7 & 0,3 & 0,6 \\
\hline
\end{tabular}

Tab. 1. Ergebnis der Wechselstrommessung am System $\mathrm{Cu}$ in $m / 100-\mathrm{Cu}\left(\mathrm{ClO}_{4}\right)_{2}$ mit $m / 100-\mathrm{HCl} \mathrm{O}$.

\begin{tabular}{|c|c|c|c|c|c|c|c|}
\hline \multirow[b]{2}{*}{$\begin{array}{l}\text { Meß- } \\
\text { frequenz } f \\
\text { in } \mathrm{kHz}\end{array}$} & \multicolumn{2}{|c|}{$\begin{array}{l}\text { Parallelwiderstand } R \\
\text { in } \Omega \mathrm{x} \mathrm{cm}^{2}\end{array}$} & \multicolumn{2}{|c|}{$\begin{array}{c}\text { Parallelkapazität } C \\
\text { in } \mu F / \mathrm{cm}^{3}\end{array}$} & \multirow{2}{*}{$\begin{array}{c}\text { Blindwider- } \\
\text { stand } 1 / \omega C \\
\text { des Parallel- } \\
\text { kondensators } \\
\text { in } \Omega \mathrm{x} \mathrm{cm}^{2}\end{array}$} & \multirow{2}{*}{$\begin{array}{c}\text { Resultierender } \\
\text { Wirkwiderstand } \\
\text { der Parallel- } \\
\text { schaltung } R_{w}= \\
R /\left(1+\omega^{2} R^{2} C^{2}\right) \\
\text { in } \Omega \times \mathrm{cm}^{2}\end{array}$} & \multirow{2}{*}{$\begin{array}{c}\text { Resultierender } \\
\text { Blindwiderstand } \\
\text { der Parallel- } \\
\text { schaltung } R_{b}= \\
R^{2} \omega C /\left(1+\omega^{2} R^{3} C^{3}\right) \\
\text { in } \Omega \times \mathrm{cm}^{2}\end{array}$} \\
\hline & $\begin{array}{c}\text { Mittel- } \\
\text { wert }\end{array}$ & $\begin{array}{l}\text { Mittlerer Fehler } \\
\text { des Mittels } \\
F_{m}=\sqrt{\frac{\Sigma^{\prime} \Delta^{2}}{n(n-1)}}\end{array}$ & $\begin{array}{l}\text { Mittel- } \\
\text { wert }\end{array}$ & $\begin{array}{l}\text { Mittlerer Fehler } \\
\text { des Mittels } \\
F_{m}=\sqrt{\frac{\Sigma \Delta^{2}}{n(n-1)}}\end{array}$ & & & \\
\hline $\begin{array}{r}1 \\
2 \\
5 \\
12 \\
16 \\
20\end{array}$ & $\begin{array}{l}22 \\
15 \\
9,2 \\
6,0 \\
5,5 \\
5,3\end{array}$ & $\begin{array}{l} \pm 0,35 \\
\pm 0,31 \\
\pm 0,4 \\
\pm 0,24 \\
\pm 0,26 \\
\pm 0,25\end{array}$ & $\begin{array}{r}11,5 \\
9,6 \\
7,7 \\
6,3 \\
5,9 \\
5,7\end{array}$ & $\begin{array}{l} \pm 0,26 \\
\pm 0,11 \\
\pm 0,3 \\
\pm 0,2 \\
\pm 0,17 \\
\pm 0,3\end{array}$ & $\begin{array}{r}11,0 \\
9,2 \\
4,2 \\
2,1 \\
1,7 \\
1,4\end{array}$ & $\begin{array}{l}6,2 \\
3,6 \\
1,6 \\
0,7 \\
0,5 \\
0,4\end{array}$ & $\begin{array}{l}9,9 \\
6,5 \\
3,5 \\
1,9 \\
1,5 \\
1,3\end{array}$ \\
\hline
\end{tabular}

Tab. 2. Ergebnis der Wechselstrommessung am System Pb/4-m. Schwefelsäure.

senheit einer praktisch kaum sichtbaren schlechtleitenden Oxydschicht mit einem Widerstand von ca. $1,5 \mathrm{Ohm} \cdot \mathrm{cm}^{2}$ immerhin ziemlich überraschend sein.

Ferner wurde eingangs darauf hingewiesen, daß auch eine Widerstandspolarisation eine gewisse Zeit zum Ausbilden und Abklingen braucht, was bisher in der Literatur nicht immer beachtet wurde. Man ist nunmehr für die beidên gemessenen Systeme in der Lage, die von der Dicke der jeweiligen Schicht unabhängige Abklingzeit nach $\tau=R \cdot C \mathrm{zu}$ berechnen. So ergibt sich für die Abklingzeit der Widerstandspolarisation beim System $\mathrm{Cu} / \mathrm{Cu}$ Ionenlösung $\tau=1,7 \cdot 10^{-5}$ sek und für das System $\mathrm{Pb} / 4-m$. Schwefelsäure $\tau=3 \cdot 10^{-5}$ sek. Es ist vielleicht kein Zufall, daß diese Abklingzeiten größenordnungsmäßig übereinstimmen mit der Belastungsdauer während einer Viertelperiode derjenigen Wechselstromfrequenz, oberhalb der die gemessenen Beträge $C$ und $R$ frequenzunabhängig werden.

Unter plausibler At.nahme einer Schichtdicke von $100 \AA ̊$ ergibt sich z. B. für die Bleisulfatschicht ein spezifischer Widerstand $\varrho=5 \cdot 10^{6} \mathrm{Ohm} \cdot \mathrm{cm}$. Für die auf der Oberfläche der Kupferelektrode angenommene Oxydschicht, unter der Annahme einer Schichtdicke von $10 \AA$, würde der spezifische Widerstand $1,5 \cdot 10^{7} \mathrm{Ohm} \cdot \mathrm{cm}$ betragen, was größenordnungsmäßig zwischen dem angegebenen spezif.Widerstand für $\mathrm{CuO}$ von $2 \cdot 10^{6} \mathrm{Ohm} \cdot \mathrm{cm}$ und dem für $\mathrm{Cu}_{2} \mathrm{O}$ zu $0,6 \cdot 10^{6}$ bis $2 \cdot 10^{7} \mathrm{Ohm} \cdot \mathrm{cm}$ liegt. Ferner muß bei so schlecht leitenden Grenzflächenschichten auf Elektroden der auf den Elektrodenabstand 0 extrapolierte Widerstand einer Leitfähigkeitszelle einen endlichen Wert besitzen, wie sich im Versuch unmittelbar bestätigen läßt. Solche Grenzflächenschichten können an einer stromlosen definierten einfachen Elektrode das chemisch-thermodynamische Gleichgewicht und das dazugehörige Galvanipotential nicht stören; ja u.U., wie bei Elektroden zweiter Art, sind sie sogar dazu sehr wesentlich. Dagegen können sie sehr wohl das Mischpotential einer stromlosen Mehrfachelektrode und vor allen Dingen die Polarisationseigenschaften einer gleichstromdurchflossenen einfachen Elektrode mitbeeinflussen. 
Soweit sich Widerstandspolarisation bemerkbar macht, ist allerdings zu beachten, daß die Dicke solcher Schichten stark abhängig ist von der Vorgeschichte und der Behandlung der Elektrode. Insbesondere kann durch Gleichstrombelastungen eine Vergrößerung bzw.Verkleinerung der Schichtdicke dieser Grenzflächenschichten erfolgen. So würde man im allgemeinen bei anodischer Belastung der Elektrode eine weitere Bildung von Oxyd und damit eine Erhöhung des Widerstandes erwarten dürfen, während bei kathodischer Belastung die Reduktion solcher Oxydschichten und damit eine Verringerung des Widerstandes erfolgen kann. Andererseits ist umgekehrt durch Absprengen von Oxydschichten beim anodischen Inlösunggehen 'von Metallionen auch eine anodische Erniedrigung und durch kathodische Bildung von Hydridschichten eine kathodische Erhöhung der
Widerstände von Grenzflächenschichten denkbar. Man kann also natürlich aus dem im unbelasteten Zustand einer Elektrode gemessenen Widerstand einer Grenzflächenschicht auch keine allzu weitgehenden Schlüsse auf die bei stärkeren Gleichstrombelastungen auftretende Gesamtpolarisation ziehen.

Anmerkung bei der Korrektur: Bei der Fortsetzung der vorstehenden Untersuchung wurde festgestellt: 1. Die Frequenz-Unabhängigkeit von $R$ und $C$ tritt auch bei der Ausdehnung der Frequenzen bis zu $100000 \mathrm{~Hz}$ auf. 2. Am System $\mathrm{Ag} / \mathrm{AgJ} / \mathrm{Ag}+$-Lösung nehmen mit zunehmender, anodisch hergestellter Schichtdicke von AgJ die frequenzunabhängigen Werte von $R$ und $C$ erwartungsgemäß zu bzw. ab, während $\tau=R \cdot C-$ außer bei den dünnsten Schichten - verhältnismäßig konstant bleibt.

Wir benützen gern die Gelegenheit, Hrn. Prof. Dr. Heinrich F a B bender als Gast und Hrn. Dipl.-Ing. $\mathrm{K}$ a r l H of fman n für wertvolle elektrotechnische Ratschläge und Hilfeleistung verbindlichst $\mathrm{zu}$ danken.

\title{
Zur Biochemie der Schilddrüsenfunktion IV': Mehlnährveränderungen der Kaninchen-Thyreoidea
}

\author{
Von Theodor Wagner-JaUREgG und Friedrich Hüter
}

Aus dem Institut für Chemotherapie „Georg-Speyer-Haus“, Frankfurt a. M.

(Z. Naturforschg. 1, 392-395 [1946]; eingegangen am 20. Mai 1946)

\begin{abstract}
Einseitige Fütterung von Kaninchen mit Weizenmehl ruft nach einigen Wochen eine histologische Schilddrüsenveränderung, ohne Vergrößerung, hervor, die als Anzeichen einer beginnenden Organschädigung zu betrachten ist. Gleichzeitige Verabreichung von C-Vitamin unterdrückt diesen Effekt, B-Vitamine oder Spuren Jod beeinflussen ihn nicht. Roggenmehlernährung ergibt normale Schilddrüsen. Die Kohlkropfnoxe wird in ihrer Wirkung durch Weißmehlfutter abgeschwächt.
\end{abstract}

$\mathrm{D}_{\mathrm{s}}^{\mathrm{i}}$ ie über einen längeren Zeitraum täglich durchgeführte Verabreichung von Präparaten mit der Schlundsonde stellt für Kaninchen eine unphysiologische, nicht ungefährliche Belastung dar. Eine andere Möglichkeit der peroralen Behandlung ist das Verfüttern von mit Wasser angeteigten und nachher getrockneten Mehlplätzchen, denen bei der Zubereitung der zu prüfende Stoff beigemengt wird. Wir stellten aber vor einigen Jahren fest, daß die während mehrerer Wochen durchgeführte einseitige Ernährung mit täglich $80 \mathrm{~g}$ Weizenmehl (Type 1050), neben Heu, nicht ohne Einfluß auf die Kaninchenschilddrüse bleibt: es kommt zwar nicht zur Vergrößerung, aber zu 1 III. Mitteilg.: F. Hü ter, diese Z. 1, 283 [1946]. einer deutlichen histologischen Veränderung des Organs, bei erniedrigtem Jodgehalt ${ }^{2}$. Die damals nur an zwei Tieren erhobenen Befunde konnten im Laufe der letzten Jahre durch weitere Beispiele bestätigt werden, wobei täglich $5 \gamma$ Jod oder $200 \gamma$ Vitamin $B_{1}$ bzw. beides zugefüttert wurde, ohne daß sich der Befund wesentlich änderte (Tab.). Im mikroskopischen Bild ist das charakteristischste Merkmal wohl die vermehrte Epithelbildung; die Follikel sind verkleinert, vielfach nur teilweise gefüllt, häufig überhaupt leer (Abb. 1 u. 2). Der Kontrollversuch mit Roggenmehlplätzchen (Kaninchen 446 und 439, Tab.) ergab, mit od sr ohne Zulage

2 Th. Wagner-Jauregg u. J. K o :h, unveröffentlicht. 